\title{
AOR
}

Selected Papers of \#AolR2019:

The $20^{\text {th }}$ Annual Conference of the

Association of Internet Researchers

Brisbane, Australia / 2-5 October 2019

\section{FROM OPEN KNOWLEDGE SHARING TO SEMI-CLOSED GROUPING: THE EVOLUTION OF ACADEMIC SOCIAL MEDIA IN CHINA}

Xiang Ren

Western Sydney University

\section{Introduction}

While China has a growingly globalised system for scientific research, its social media system has long been separated from the rest of the world as Facebook, Twitter and the like are blocked in China. On the other hand, Chinese technology companies have developed alternatives with vast amounts of users like WeChat and Weibo, which create a different system of academic social media, with different evolutionary trajectories and political economy (Yu, Asur, and Huberman, 2001).

This paper reviews the evolution of Chinese academic social media in the past twenty years or so, with an analytical focus of trust and openness. It examines and compares the communicative models in scientific blog, Weibo and WeChat, and explores how academic social media co-evolve with academics' changing demands, as well as broad social and institutional contexts in China.

This work identifies transformative changes of Chinese academic social media practices, particularly the shifting focus from open sharing of knowledge in public sphere to semi-closed and semi-public grouping based on acquaintance networking. While Chinese academics believe acquaintance grouping enables more reliable and rewarding communications, this raises issues regarding a "closed" and "exclusive" approach to building trust in scholarly/scientific communications. Inspired by the concept of "knowledge club" (Potts, et al., 2017), this paper understands the evolution of Chinese academic social media as a process of "clubization" of digital knowledge systems and further discusses the reasons and impact in the Chinese contexts.

\section{Methods, findings and arguments}

This research employs multiple methods and combines research data collected at Suggested Citation (APA): Ren, X. (2019, October 2-5). From open knowledge sharing to semi-closed grouping: The evolution of academic social media in China. Paper presented at AolR 2019: The $20^{\text {th }}$ Annual Conference of the Association of Internet Researchers. Brisbane, Australia: AolR. Retrieved from http://spir.aoir.org. 
different periods of time in order to understand the evolution of Chinese academic social media from a multidimensional and historical perspective. It draws on over 20 in-depth interviews conducted during 2010-9 across different periods of social media evolution, 8 years' participatory observation in Science Net Blog, Sina Weibo and WeChat following approximately 200 popular social media accounts and groups in scientific and scholarly communications, and document analysis and discourse analysis of corporate files, media reports, and online discussions.

It categorises the evolution of Chinese academic social media into three stages, as represented by three influential platforms, namely, blogging (Science Net Blog), microblogging (Sina Weibo) and social grouping (WeChat).

- Like academics elsewhere in the world, the Chinese scholars began to share research, exchange ideas, engage discussions, and connect peers in scientific BBS portals in the 1990s. However, academic social media have not become an influential medium for informal scholarly communication until early 2000s when scientific blogging gained momentum in China, in particular, with the rise of Science Net blog. The emergent scientific blogosphere attracted both leading academics and early/mid-career scholars and started to challenge the established formal scientific communication system.

- This transformation was further advanced by micro-blogging. Launched in China in 2009 , Sina Weibo soon became an extremely popular social media platform. Weibo offers unprecedented interactivity, inclusiveness and connectedness in informal scholarly communication, especially enabling academics to effectively engage with general publics.

- Two years after Weibo's launch, WeChat prevailed and soon became an infrastructural platform of China's digital economy and society (Zhang, Li, Wu, and $\mathrm{Li}, 2017$ ). The new communicative models and social functions of WeChat, based on acquaintance networking and semi-public grouping, are redefining boundaries between public and private communications, in which, academics mostly communicate with selected group members and use social media for extending and enhancing real-world networks rather than contributing to virtual public sphere.

The paper argues that, the changes of academic social media practices suggest a coevolution between the changing demands of Chinese academics and the communication models of social media. While sharing, publishing and networking are basic functions of all forms of social media, different social media platforms have different priorities at different evolutionary stages (van Dijck, 2013). Blogs like Science Net serve as a public sphere within academia, along with relevant functions like a virtual water cooler and open access repository, which, however, have limited direct public engagement. Micro-blogging offers a more efficient and interactive way for scholarly communication and academic outreach, and especially extends the scope beyond academic ivory tower. However, the inclusiveness of Weibo is a double-edged sword, as controversies exist in the quality, trust and efficacy of scholarly communication, as well as the difficulties in China's tightly censored Internet. WeChat seems to offer a solution through semi-public and semi-closed grouping, which, however, is exclusive by nature, given the maximum members for a WeChat group is only 500 . 


\section{Discussion and Conclusion}

The evolution of academic social media suggests several important transitions: from access to usage, from scale to quality, from sharing to grouping, and from inclusive and anonymous virtual communities to selective acquaintance networks. Potts et al propose an economic model of "knowledge club" to redefine journal publishing, where members form self-constituted groups, endeavouring to create new knowledge (Potts, et al., 2017). For "knowledge clubs", balancing the positive externalities of commons against the negative externalities of crowding in knowledge communications is key. The concept of "knowledge club" offers a perspective to explain the evolutionary changes of academic social media in China, particularly the decline of Weibo's public sphere and the rise of WeChat groups. However, it raises new issues regarding trust and openness.

Trust in content, participants, platforms, and regulations is in the centre of social media transformations. Social media is usually theorised as an "open" sphere. Interestingly, the Chinese social media evolution suggests a more "closed", or "semi-closed" approach to building trust. For example, instead of universal open access to knowledge as a public good, many WeChat groups are "by-invitation only" where like-minded people, acquaintances, or people with similar levels of expertise share knowledge as a club good. Further, social media are usually viewed as disruptive technologies, bypassing traditional institutions (Evens, 2013), which tend to democratise knowledge systems. However, through acquaintance networking or "knowledge clubs", social and symbolic capitals of the real world institutions are increasingly important for building reputation and influence in the virtual social media world, which, re-institutionalises digital sphere and replicates the power structure of the established academic systems.

This paper hopes to further the discussions on trust in the digital media systems through a focus of Chinese academic social media and the perspective of trust and openness. For example, does the semi-public WeChat grouping suggests a trend of clubisation of social media and knowledge systems in China (and beyond)? How can we balance the closed or semi-closed models like "WeChat clubs" and open public sphere?

\section{References}

Evens, T. (2013). Platform leadership in online broadcasting markets. In Handbook of Social Media Management (pp. 477-491). Springer, Berlin, Heidelberg.

Potts, J., Hartley, J., Montgomery, L., Neylon, C., \& Rennie, E. (2017). A journal is a club: a new economic model for scholarly publishing. Prometheus, 35(1), 75-92.

van Dijck, J. (2013). The Culture of Connectivity: A Critical History of Social Media: OUP USA.

Yu, L., Asur, S., \& Huberman, B. A. (2011). What trends in Chinese social media. arXiv preprint arXiv:1107.3522

Zhang, C. B., Li, Y. N., Wu, B., \& Li, D. J. (2017). How WeChat can retain users: Roles of network externalities, social interaction ties, and perceived values in building continuance intention. Computers in Human Behavior, 69, 284-293. 